\title{
The Impacts of Satellite Remotely Sensed Winds and Total Precipitable Vapour in WRF Tropical Cyclone Track Forecasts
}

\author{
Diandong Ren \\ ASDI, Curtin University, P.O. Box U1987, Perth, WA 6845, Australia \\ Correspondence should be addressed to Diandong Ren; rendianyun@gmail.com
}

Received 6 July 2016; Accepted 25 September 2016

Academic Editor: Anthony R. Lupo

Copyright ( 2016 Diandong Ren. This is an open access article distributed under the Creative Commons Attribution License, which permits unrestricted use, distribution, and reproduction in any medium, provided the original work is properly cited.

\begin{abstract}
This study assesses the impact assimilating the scatterometer near-surface wind observations and total precipitable water from the SSMI, into WRF on genesis and track forecasting of four tropical cyclones (TCs). These TCs are selected to be representative of different intensity categories and basins. Impact is via a series of data denial experiments that systematically exclude the remote sensed information. Compared with the control case, in which only the final analysis atmospheric variables are used to initialize and provide the lateral boundary conditions, the data assimilation runs performed consistently better, but with very different skill levels for the different TCs. Eliassen-Palm flux analyses are employed. It is confirmed that if a polar orbital satellite footprint passes over the TC's critical genesis region, the forecast will profit most from assimilating the remotely sensed information. If the critical genesis region lies within an interorbital gap then, regardless of how strong the TC later becomes (e.g., Katrina 2005), the improvement from assimilating near-surface winds and total precipitable water in the model prediction is severely limited. This underpins the need for a synergy of data from different scatterometers/radiometers. Other approaches are suggested to improve the accuracy in the prediction of TC genesis and tracks.
\end{abstract}

\section{Introduction}

Weather and climate hazards are causing increasingly more damage [1]. In recent years, there has been a sharp rise in human and property loss from weather- and climate-related disasters. Tropical cyclones (TCs), as a representative of highimpact weather, are major causes of these disasters, with recent storms such as Hurricane Sandy in northeast USA in 2012 [2] and Typhoon Haiyan in the Philippines in 2013 [3, 4]. It is important to note that TCs also are beneficial as their accompanying heavy rainfall is the major source of fresh water for many tropical countries [5-7]. Given both their negative and positive impacts more accurate predictions are required for TC tracks and intensity, for supporting emergency management and mitigation of impacts and economic losses. This study focuses on improving TC track forecasting by further assimilating remotely sensed, near-surface (e.g., $10 \mathrm{~m}$ above sea level) oceanic wind fields and total precipitable water (TPW). Over the oceans, aircraft measurements are less dense than those over land, and surface synoptic observations (SYNOPs) and radiosondes are generally not available. As a consequence, satellite data, which maintains continual coverage of the tropics in space and time, have become increasingly important for improving TC forecasting.

Fortuitously, the satellite era provides satellite winds (surface winds, cloud-drift winds, and moisture-drift winds) and atmospheric column radiance that reflects the atmospheric column water content. IR and microwave sounders provided water vapour profiles (e.g., from many polar orbiters such as the Aqua A-Train and NOAA POES). These information sources have proved to be extremely valuable in improving numerical weather prediction (NWP) model skill [8-10]. In tropical regions, information on winds is more efficient at recovering equatorial waves than mass fields [11].

Satellite observations play a critical role in NWP systems $[10,12-15]$. With better understanding of the large-scale processes that control TC dynamics, there have been considerable advances in TC prediction, especially with the steady improvement of mesoscale modelling systems and the advent of increasingly powerful computing resources to run the models. Accurate prediction of TC activity still poses challenges [16]. For example, the operational version of the Global 
Forecast System (GFS) models failed to predict the leftward (west) turning of the extremely damaging Hurricane Sandy (2012), after it left the Caribbean. By a series of denial experiments, Mcnally et al. [17] proved that assimilating remote sensing data, especially polar orbiters, were critical in forecasting Sandy's abrupt leftward motion. Zhu et al. [18] further confirm this assertion. In this study, satellite retrieval products from polar-orbiting satellites are assimilated by an operational NWP system: the nonhydrostatic Advanced Research Weather Research and Forecasting model (ARW-WRF [19]), to assess the improvements in the forecasting skill of tropical cyclone position. To draw conclusions that are generally applicable over the global basins, we intentionally selected four tropical cyclones over the two largest TC basins: Ketsana (2003) and Megi (2010), in the North Western Pacific basin, and Katrina (2005) and Maria (2011) in the Atlantic basin. These TCs also contrast in strength and severity. For example, although formed in the same TC basin, Katrina reached Category 5 and Maria reached only Category 1 . Two of the systems made landfall and two of them were oceanic only.

Here, an assessment is made of the added value of assimilating surface winds from QuikSCAT scatterometer (hereafter, QSCAT) and the Advanced Scatterometer (ASCAT, http://www.remss.com/missions/ascat [20]) and total precipitable water from the Special Sensor Microwave Imager (SSMI [21]). Data denial experiments were conducted using WRF with 34 vertical levels (up to $50 \mathrm{hPa}$ and with a horizontal resolution of $5 \mathrm{~km}$ ). The data assimilation system cycled for two days and the forecasts followed. For each TC, three simulations were performed. In the first (control) simulation, no additional satellite data were assimilated (except those conventional pieces of information already in the analyses), and only NCEP Global Forecast System (GFS) 1-degree data were used to initialize WRF, which was run for 4 days (96-hour prediction cycles starting at 00 UTC and 12 UTC each day). In the second experiment, SSMI-measured total precipitable water was assimilated and the model was run for four days. In the third experiment, near-surface wind vectors from QSCAT were additionally assimilated.

This paper is organized as follows: Section 2 describes the important characteristics of the SSM/I and QSCAT/ASCAT satellite observations to be assimilated by WRF and the International Best Track Archive for Climate Stewardship (IBTrACS, [22]; http://www.ncdc.noaa.gov/oa/ibtracs) for verification of WRF model improvements. A brief description of the WRF configuration used, the WRF 3Dvar data assimilation system (WRF-DA), the quality control procedures, and the case studies is given in Sections 3 and 4. Section 5 assesses the added value of SSM/I and ASCAT data over the traditional approach (i.e., a control run utilising only large-scale reanalysis data), by comparing a series of denial experiments that systematically include these two types of satellite products. Section 6 provides a summary and draws conclusions that are basin-independent.

\section{Data}

Here, the radiometer observations of oceanic (near)surface $(10 \mathrm{~m}$ asl) wind speeds and total precipitable water (TPW) from the Special Sensor Microwave Imager (SSM/I, http:// www.remss.com/missions/ssmi) were assimilated into the WRF-DA 3Dvar system. These data have been available from the Defense Meteorological Satellite Program (DMSP) since 1987 (http://www.remss.com/). This study used the F13-F16 series available publically on the Remote Sensing Systems (RSS) website (ftp://ftp.ssmi.com/ssmi/ [21]). Orbital observations within our simulation domain (Figure 1) were used. Total precipitable water from SSMI was also assimilated (Figure 2).

Because the apparent interorbital gap and information redundancy are critical for successful minimization of the cost function, the QSCAT wind vectors also were assimilated. The level 2 swath data are available from the RSS website (http://www.remss.com/missions/qscat). Two of the TC cases in this study (Katrina 2005 and Ketsana 2003) have data from QSCAT in its fully operational period before November 2009. For TCs Megi (2010) and Maria (2011), the level 2 along-track data $(12.5 \mathrm{~km}$ resolution) from Metop-A ASCAT are used. QSCAT and now its replacement, ASCAT, provide highresolution observations over the TC basins of interest to this study. More importantly, sea-surface wind speed and direction data, obtained using microwave observations, are available under a wide range of weather conditions over the Earth's oceans. For example, EUMETSAT's ASCAT, as a C-band scatterometer, can provide wind observations even under light rainfall conditions. This is very important for TC studies because clouds are prevalent within and around Typhoons and hurricanes [23]. The highly valuable QSCAT measurements ceased in 2009, but the ASCAT data is available since then.

TC best track observations were obtained from the IBTrACS, which provides the latitude and longitude of the center of circulation, the maximum sustained winds, and the minimum sea level central pressure values, every 6 hours. IBTrACS data were used to verify improvements in the track forecasts. In addition, the ERA-Interim ( $\sim 79 \mathrm{~km}$ resolution) reanalyses were used to assess large-scale performance such as the forecast skill for winds, temperature, and water vapour content [24]. The assessment was based on a comparison of the forecasts with the ERA-Interim reanalyses.

\section{The Data Assimilation Procedure and Quality Control}

The assimilation of satellite data has become widespread and has been an active research area over the past three decades. Satellite passive infrared and microwave radiance observations are routinely assimilated into NWP systems at the National Center for Environmental Prediction (NCEP) and the European Centre of Medium-Range Weather Forecasts $[10,25,26]$. Significant improvements in NWP global forecasts, particularly over the southern hemisphere, have been achieved from direct radiance assimilations, especially microwaves (e.g., Advanced Microwave Sounding Unit-A (AMSU-A) and the Microwave Humidity Sounder (MHS)).

In prediction mode, assimilation of available remotely sensed data is a critical step in prescribing accurately the initial conditions and thereby improving TC track predictions. 


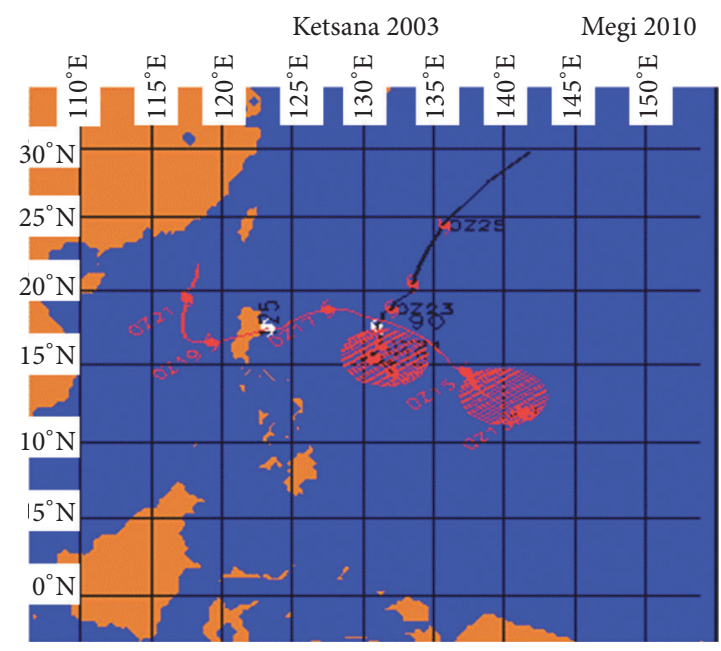

(a)

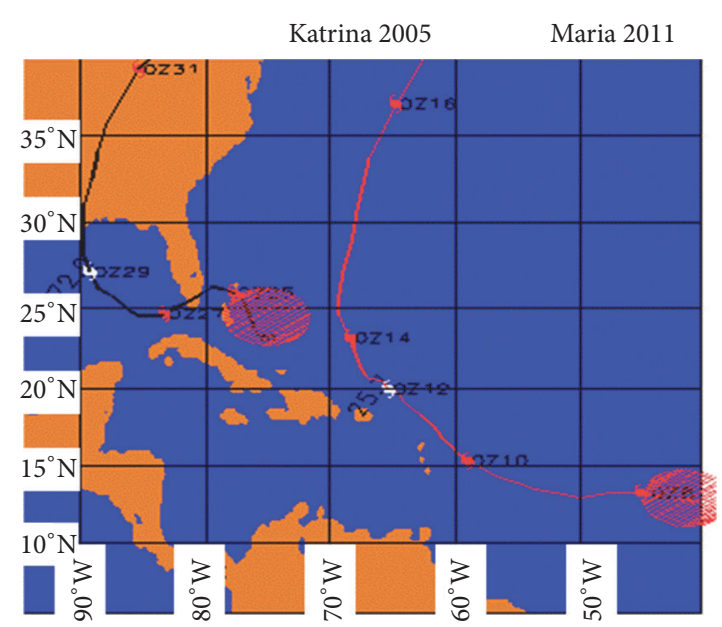

(b)

FIGURE 1: Best tracks of the tropical cyclones investigated: Katrina (2005) from 00 UTC, 22 August 2005, to 00 UTC 31, August 2005; Maria (2011) from 00 UTC, 7 September, to 00 UTC, 14 September; Megi (2010) from 00 UTC, 12 October, to 00 UTC, 23 October; and Ketsana (2003) from 00 UTC, 18 October to 00 UTC, 26 October. The storm intensity is indicated at $12 \mathrm{hr}$ intervals at 00 UTC and 12 UTC, respectively. Time of attaining maximum strength is shown (see white marker). E-P fluxes are estimated within the red hatched areas.

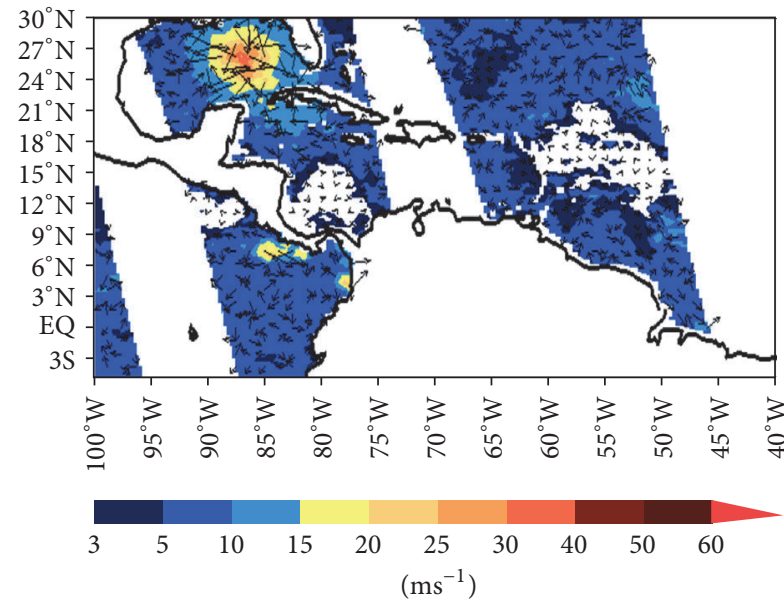

(a)

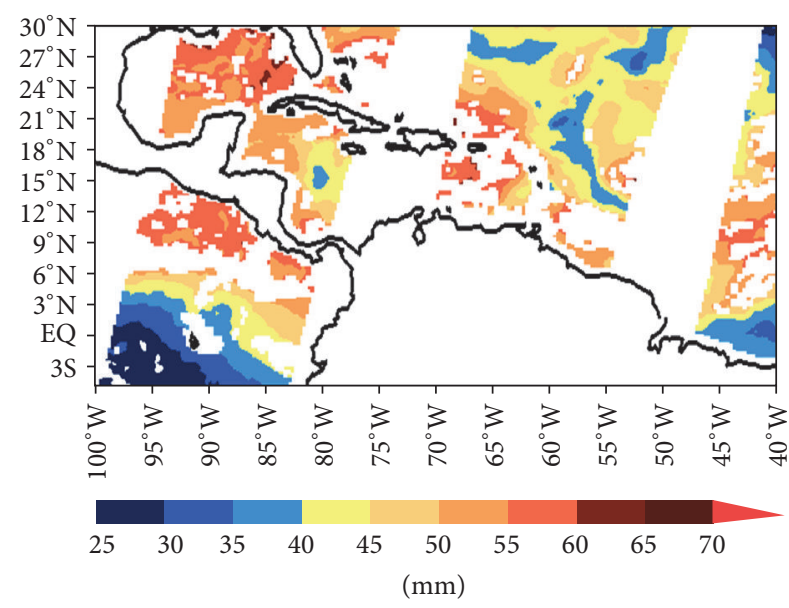

(b)

FIGURE 2: Spatial distribution of 0.25-degree QSCAT data (a) near-surface wind vectors ( $\mathrm{m} / \mathrm{s}$ ) and (b) SSM/I measured total precipitable water $(\mathrm{mm})$ in a 6-hr window centered on 12 UTC on 24 August 2005. In (a) those vectors close to sensor saturation (30 m/s) are filtered out in the quality control stage.

The WRF-DA 3Dvar system developed at the National Center for Atmospheric Research (NCAR) is used for the assimilation of remotely sensed surface wind vectors and total precipitable water. The aim of variational data assimilation process is to obtain an optimal estimate of the true atmospheric state at a desired analysis time through iterative minimization of the prescribed cost function $J(x)$ [27]:

$$
\begin{aligned}
J(x)= & \frac{1}{2}\left(x-x_{b}\right)^{T} B^{-1}\left(x-x_{b}\right) \\
& +\frac{1}{2}(y-H(x))^{T} R^{-1}(y-H(x)),
\end{aligned}
$$

where $x$ is the atmospheric state vector, $x_{b}$ is the background state (usually a short-range forecast), $H$ is the nonlinear observational operator, and $y$ is the observation vector. For satellite remote sensing, $H$ is responsible for transforming model parameters/analysis to be comparable with observations. For example, atmospheric radiative transfer models (RTMs) convert atmospheric parameters to be comparable with satellite observations of top-of-atmosphere spectral radiance (e.g., the Community Radiative Transfer Model, CRTM, https:/groups.ssec.wisc.edu/groups/itwg/rtsp and the Radiative Transfer code for TOVS, RTTOV [28]). In this study, the wind vectors and precipitable water are already 
provided in model comparable format or, alternatively, are satellite-derived variables and then $H$ is simply an interpolation operator [29].

$B$ and $R$, respectively, are the background and observation error covariance matrices. They are directly associated with the data source (e.g., the source that provides the remote sensing data should also provide the corresponding $R$ ) and need to be properly specified and (for in situ measurements) at each location. For satellite-derived observations, one uniform value is assigned (to $R$ ) for the spatial field. The spatial resolutions for observations are chosen to be twice the model resolution, minimizing the spatial correlations among observations.

The background error statistics are estimated using Parrish and Derb's [30] method. A set of cold-start forecasts was initiated from GFS analyses at 00 UTC and 12 UTC every day from one month leading to the TC events. The differences in the 00 UTC and 12 UTC forests are then used to derive the background error statistics. The background error statistics are computed for the above-described domains. The identical cycling data assimilation and forecast configuration are employed for other selected TCs. For example, for Katrina (2005), only the analysis time starts from 00 UTC 23 August to 2 September 2005. In these experiments, data from within $\pm 3 \mathrm{hr}$ of analysis times were used and assumed to be valid at the reanalysis times.

If an iterative solution for $x$ can be found by minimizing (1), the result represents a minimum variance estimate of the true atmospheric state, given the background $x_{b}$ and observations $y$, as well as $B$ and $R$ [29]. In this proposed research, whenever an observed wind vector is available, a 3Dvar analysis is performed in order to optimize the initial conditions and thereby permit forward predictions to be made based on the adjusted initial conditions. In practice, after adjustments to initial conditions, inconsistency may arise at the boundary and proper adjustments (e.g., smoothing) are needed at the boundaries.

This study uses satellite-retrieved products but quality control procedures are equally important. Our assimilation operations are within the first three days of the TC life cycle after being identified (in IBTrACS records) because of one quality control issue with respect to the QSCAT/ASCAT data. The saturation value for wind speed is $30 \mathrm{~m} / \mathrm{s}$. After the storm reaches Category 4 , there are many locations where the actual wind speed exceeds this saturation value. Separate (OSSE) experiments indicate that including of these erroneous saturation values can dramatically reduce TC predictive skill.

Operational NWPs use frequent updating cycles (every 12 or even 6 hours), with the predictions out to 72 hours or at most to one week. For TC predictions, the assimilation of the ASCAT/QSCAT measurements for the first three days after the formation of the tropical depression is free of wind speed saturation (i.e., reaching the threshold value of $30 \mathrm{~m} / \mathrm{s}$ ). After the TC reaches Category 4, a significant portion of the TC $\left(\sim 100 \mathrm{~km}^{2}\right)$ surrounding the inner core [31] achieves sustained wind speeds greater than the QSCAT/ASCAT threshold values. Quality control is necessary to eliminate wind vectors in this region, as they inhibit improvements and degrade the data assimilation procedure. Assimilating the remaining wind vectors provides important information and increases the TC predictive skill. The retrieval experiments performed in this study assimilate remote sensing data at the early development stage of TCs and, even for the central core regions, few winds reach the QSCAT/ASCAT threshold values.

\section{Numerical Experiments}

For each TC, the simulation is initiated two days earlier than the IBTrACS start time and is run until one day after landfall (or ten days for the two life-time oceanic TCs Ketsana 2003 and Maria 2011). To identify the relevant mechanisms (noncase sensitive, universally applicable improvements in predicting TC activities), four TCs were assessed, covering the four major global basins. Three cycles were performed (one SSMI and two QSCAT), each of 72-hour forecast length and with innovation time levels at 12 UTC for SSM/I and 06 UTC for QSCAT/ASCAT (see Figure 3, for Megi 2010). These innovation time levels were chosen because the 6-hr wind centered on them provides the best overlap with the satellite pass. The assimilation-forecast cycle is repeated every two days. Using Ketsana 2003 as an example, the procedure is as follows.

Ketsana 2003 formed in a monsoon trough between Luzon and Guam during a positive phase of the MJO. It was first identified on 12 UTC 18 October as a tropical disturbance, approximately $900 \mathrm{~km}$ southeast of the Philippines. It attained wind speeds just below Typhoon status the next day. On 20 October, it intensified rapidly into a Typhoon and developed an eye. On 22 October, JTWC estimated Ketsana's peak winds at $165 \mathrm{~km} / \mathrm{hr}$. Ketsana continued moving northwards into westerlies and gradually weakened. On 26 October, it became an extratropical cyclone and dissipated the next day. The life cycles of the other three TCs are in Table 1.

The 6-hour cycling forecast-analysis experiments are carried out for all four selected TCs. For Ketsana, the data assimilation period begins at 06 UTC on 16 October 2003. The background field in the first analysis at 06 UTC is provided by a forecast of the WRF run initiated from NCEP GFS $1 \times 1$ degree analysis at 00 UTC on 16 October, with observed SST at lower boundary. For the ensuing cycles, the background fields are the 6-hour WRF forecasts from previous cycle. The lateral boundary conditions for the WRF forecasts are also provided by the GFS analyses at 6-hr intervals.

The nonhydrostatic WRF 3.3.1 with the ARW dynamical core is employed to simulate the processes leading to the cyclogenesis and intensification of the selected TCs. This version of WRF is further modified by the implementation of the effect of salinity on surface latent heat fluxes [32]. Three nested domains are set with horizontal spatial resolutions of $27 / 9 / 3 \mathrm{~km}$ with one-way nest option, and the number of gridpoints (mesh sizes, using Ketsana 2003 as an example) is $198 \times 154(\mathrm{D} 1), 295 \times 232(\mathrm{D} 2)$, and $436 \times 346(\mathrm{D} 3)$, respectively. In the vertical direction, there are 35 layers with the model top at $50 \mathrm{hPa}$. The following model physics schemes are used [32]: the WRF single-moment 6-class microphysics scheme with graupel (WSM6 [33]) and the Yonsei University 


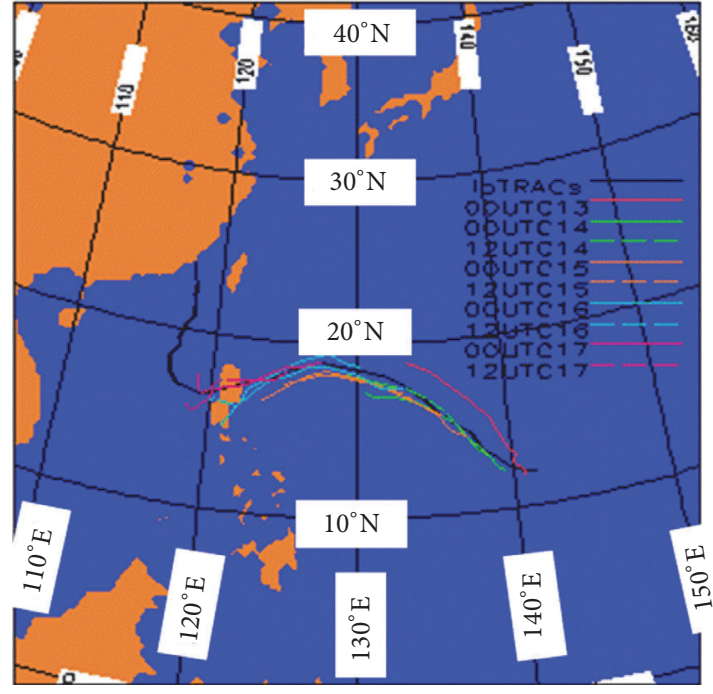

(a)

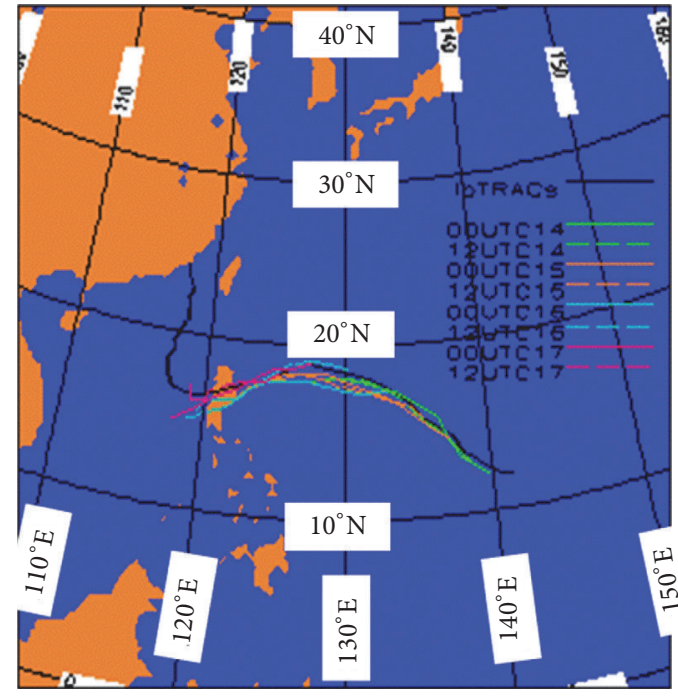

(b)

FIGURE 3: 72-hour prediction cycles of Megi (2010) (a) and the corresponding assimilation cycles (b) for assimilating SSM/I at 12 UTC. The reduction of track error from $50 \mathrm{~km}$ down to $20 \mathrm{~km}$ is very satisfactory considering that the satellite data are of similar spatial resolution.

TABLE 1: Life cycles of the TCs investigated.

\begin{tabular}{lccccc}
\hline TC names & Date identified & Tropical Depression (TD) & Tropical Storm (TS) & TC status achieved & Date diminishing \\
\hline Ketsana 2003 & Oct. 18, 2013 & Oct. 18 & Oct. 19 & Oct. 20-23 & Oct. 26 \\
Katrina 2005 & Aug. 23, 2005 & Aug. 23 & Aug. 24 & Aug. 25-29 & Aug. 31 \\
Megi 2010 & Oct. 12, 2010 & Oct. 13 & Oct. 14 & Oct. 15-17 & Oct. 23 \\
Maria 2011 & Sept. 7, 2011 & Sept. 14 & Sept. 15 & Sept. 16 & Sept. 16 \\
\hline
\end{tabular}

Planetary boundary layer (YSU-PBL) scheme [34]; the modified version of Kain and Fritsch cumulus parameterization scheme (KF_Eta [35]) is used in the outer two domains (D1 and D2), whereas only explicit moisture calculation is used for D3. Radiation is treated using the RRTM long-wave scheme, a spectral-band radiative transfer model using the correlated K-method [36], whereas shortwave radiation was parameterized using Dudhia's 1989 [37] scheme (or the MM5 shortwave scheme). At the surface, the NOAH land surface model [38] was used with United States Geological Survey (USGS) land use data of 30 arc seconds' resolution (for D3).

The WRF run initialized at 00 UTC 16 October 2003 and integrated 240 hours until 00 UTC 26 October 2003. The model's initial and lateral conditions are taken from the NCEP data with outermost lateral boundary conditions updated every 6 hours, and the SST products are interpolated and used to update the sea-surface boundary conditions. The "operational setting" of WRF is less skillful in tropical region. For example, it cannot simulate the unusual path and intensification of Hurricane Sandy in October 2012 [16]. To improve the performance of WRF in the tropical zone, the WRF-DA (3.3.1) are used to assimilate QSCAT oceanic vector winds available at 06 UTC on 16 October and 06 UTC on 17 October and SSM/I oceanic surface wind speed and total precipitable water (TPW) available at 12 UTC on 17 October, to improve the initial conditions. These twice daily swath
QSCAT and SSM/I data are extracted from the RSS data archive with $\sim 12.5 \mathrm{~km}$ resolution for QSCAT and 0.25 degrees for SSM/I.

Four prediction experiments are designed to test the performance of data assimilation: (1) without assimilating QSCAT near-surface winds or SSM/I TPW (i.e., contains information from SSM/I winds and remote sensing information already in GFS; CNTRLs in Table 2), (2) assimilating QSCAT at 06 UTC on 16 October (i.e., Q's in Table 2), (3) further assimilating QSCAT at 06 UTC on 17 October based on experiment 2 (QS's), and (4) further assimilating SSMI at 12 UTC on 17 October based on experiment 3 (QSQ's). These experiments are designed to examine the impact of assimilating observed near-surface winds and total precipitable water on analyses and the subsequent forecasts. To evaluate more objectively the gain from data assimilation, the root mean squared error (rmse) of the tracks from CNTRL runs $\left(r_{0}\right)$ are compared with track rmse from the three data assimilation cycles. The rmse is defined as the departure from corresponding best track values, with all data grids in the model cycle equally accounted. For example, there are ten prediction cycles for Megi 2010, at 00 UTC and 12 UTC of each day from 13 October to 17 October (curves of different colors in Figure 3). Differences of analyses among the four experiments are also intercompared to gain insight into the impact of data redundancy. Table 3 lists the rmse (against the IBTrACS data 
TABLE 2: Design of experiments and satellite-derived products they assimilated.

\begin{tabular}{lcccc}
\hline TC names & $\begin{array}{c}\text { No additional assimilation } \\
\text { except that already in GFS } \\
\text { (EXP1) }\end{array}$ & $\begin{array}{c}\text { One QSCAT innovation } \\
\text { at 06 UTC, Oct. 16 } \\
\text { (EXP2) }\end{array}$ & $\begin{array}{c}\text { One QSCAT (06 UTC) and one } \\
\text { set of SSMI date 6 hours later (12 } \\
\text { UTC Oct. 16) (EXP3) }\end{array}$ & $\begin{array}{c}\text { Two QSCAT innovations (06 } \\
\text { UTC, Oct. 16 and 17) plus one } \\
\text { SSMnovation (12 UTC, Oct. } \\
16)(\text { EXP4) }\end{array}$ \\
\hline Ketsana 2003 & KeCNTRL & KeQ & KeQS & KeQSQ \\
Katrina 2005 & KaCNTRL & KaQ & KaQS & KaQSQ \\
Megi 2010 & MeCNTRL & MeQ & MeQS & MeQSQ \\
Maria 2011 & MaCNTRL & MaQ & MaQS & MaQSQ \\
\hline
\end{tabular}

TABLE 3: Improvements in TC intensity $\left(V_{\max }\right)$ and location using the various assimilations described in Table 2.

\begin{tabular}{lccc}
\hline & $\Delta V_{\max }$ & $\Delta P_{\min }(\mathrm{hPa})$ & $\Delta$ Lat $/ \Delta$ Long $(\mathrm{km})$ \\
\hline KeCNTRL & 6 & 21.0 & $18 / 100$ \\
KeQ & 4.5 & 20 & $18 / 76$ \\
KeQS & 3.0 & 17 & $15 / 50$ \\
KeQSQ & 2.85 & 17.2 & $12 / 45$ \\
KaCNTRL & 4 & 40.3 & $86.4 / 132.2$ \\
KaQ & 4 & 23.0 & $71.1 / 102.2$ \\
KaQS & 4 & 39.7 & $115.2 / 100.9$ \\
KaQSQ & 4 & 40.0 & $112.5 / 102.2$ \\
MeCNTRL & 12 & 21.8 & $42.0 / 66.0$ \\
MeQ & 10 & 17 & $35 / 45$ \\
MeQS & 7 & 14 & $23 / 33$ \\
MeQSQ & 5 & 12 & $20 / 30$ \\
MaCNTRL & 3.6 & 30.0 & $68 / 84$ \\
MaQ & 3.5 & 25 & $60 / 81$ \\
MaQS & 3.1 & 23 & $58 / 78$ \\
MaQSQ & 2.9 & 20 & $55 / 75$ \\
\hline
\end{tabular}

within each TC's life cycle) in simulated TC properties, with increasing information content to be assimilated.

\section{Results}

The forecast skill for wind, temperature, and humidity was assessed by comparing the forecasts to NCEP/NCAR reanalyses [39] and conventional observations. The storm centers' position, minimum sea level pressure, and absolute maximum wind speed data are all comparable to those from the corresponding IBTrACS.

The rmse of vertical profiles of the 60-hour forecasts for temperatures, wind speeds, and specific humidities, when compared with NCEP/NCAR reanalyses (averaged over all cycle times), are calculated for Ketsana, Katrina, Megi, and Maria, respectively. The forecast accuracy for wind, temperature, and water vapour content was promising, with wind biases generally less than $3 \mathrm{~m} / \mathrm{s}$, temperatures less than $0.7 \mathrm{~K}$, and specific humidity less than $2 \mathrm{~g} / \mathrm{kg}$.

Precipitation is one of the most challenging fields for NWP models to predict, both the patterns and intensities. Output from D1 of Ketsana and Katrina indicates that the primary rain-band is to the right (east) of the storm center. This can be explained by quasi-geostrophic theory, because the sector has a lifting mechanism, associated with the positive tendency of cyclonic vorticity and the supply of vapour from the ocean surface winds, which blow from a relatively warm ocean surface to a colder ocean surface. Outputs from the innermost domain (D3s) reveal more details about eyewall replacement processes and the evolution of smaller scale convective clusters, in the form of mesovortices. Vortex Rossby wave activity can explain the simulated phenomena [4042]. To verify the model simulated precipitation, the Tropical Rainfall Measuring Mission (TRMM), a joint mission designed to monitor and study tropical rainfall, 3B42RT grid precipitation products are used (ftp://trmmopen.gsfc.nasa .gov/pub/merged/3B42RT/). Ren et al. [32] compared TRMM measured and WRF simulated Katrina precipitation rates $(\mathrm{mm} / \mathrm{hr})$ at three different times; 18 UTC on 27 August, 15 UTC on 28 August and 03 UTC on 29 August, 2005. It was found that the precipitation was asymmetrically distributed around the eye and the areas of strongest precipitation, located in clusters, can be up to $200 \mathrm{~km}$ from the storm center. In these simulations, the patterns derived from the radar observations are well-captured by the model simulations. However, quantitative precipitations from WRF simulated totals are stronger than radar observations and also are somewhat more concentrated. The other three TCs are qualitatively similar. For example, within their life cycles, the shape of eyes transitions from being less circular to more circular (developing stage) then decays to a less circular shape. The decay occurs primarily because the surface supply of moisture is greatly diminished, either because of the proximity of the land surface or from the TC steering to higher latitudes that cannot supply such a thermal engine. Moreover, through surface layer friction, TC also significantly transfers energy vertically upward.

Done et al. [43] noted that ARW, on a $4-\mathrm{km}$ or finer horizontal resolution, may produce the detailed structure of the TCs such as distribution of maximum winds within the inner core and eyewall regions. For the WRF simulations over D3 $(\sim 3 \mathrm{~km})$, the maximum wind speeds are generally less than the best track values by as much as $20 \mathrm{~m} / \mathrm{s}$ (for Katrina 2005), even though the minimum TC sea level pressure values are comparable. Large discrepancies were evident primarily during the rapid acceleration stage of the storm's total kinetic energy (as defined in Ren et al. [32]). The minimum sea level pressure values in the forecasts also were significantly larger than the observations. For example, for Katrina 2005, the 
discrepancy is as large as $37 \mathrm{hPa}$. Although the predicted tracks are reasonably close (on the same order as the coarsest grid resolution of $\sim 100 \mathrm{~km}$ ), there are significant phase lags or leads as the numerical simulation evolves faster than observed. Further, reducing the innermost domain's resolution has a limited effect on reducing the prediction-observation discrepancies. Thus, this is more than a simple resolution dependence of the forecast. Without further improvements to the physical parameterization, it is difficult to further improve the forecast skill of both tracks and intensity.

This study primarily is focused on numerical forecasting of TC positions/tracks. TC motion is primarily steered by advection of the TC under the influence of the surrounding environmental flow [44-47]. While there are other mechanisms which can affect the motion of TC, the environmental "steering" [48] flow generally is the dominant contribution, especially for steady westward stage of TC motion [49]. This explains why TCs move around the subtropical high pressure systems, such as the Bermuda High. However, the interaction of TC with its complex and dynamically evolving environment and the resultant steering has been a subject of extensive modelling research for many years [49-53]. By providing four-dimensional, dynamically consistent highresolution atmospheric fields, NWP simulations provide insight into the relative importance of various synoptic-scale features of the TC environment. However, due to the data paucity over the oceans, it still is challenging for NWP models to accurately simulate the TC circulation features, especially during the early stages of development.

Numerical models currently provide the most accurate track projections also for investigating the underlying reasons for a particular track. Here the focus is not on the mechanisms controlling TC motion but on the impact on track prediction improvements from the assimilation of remote sensing data. For Katrina, the simulated control track is to the east of IBTrACS observations by $\sim 0.4$ degrees at most (Figure $2(\mathrm{a})$ ), and the additional data aligns the track closer to the observed track. $r_{0}$ (CNTRL run) values for TC Maria and TC Megi are both reasonably small (0.6 degree). However, for Ketsana, the predicted track is to the west by as much as 1.2 degrees. These are the baseline results for WRF's performance driven only by the boundary conditions from the NCEP GFS. From Table 3, all assimilated information has an impact and experiments QSQs are closest to IBTrACS. The improvement, as measured by the ratio to $r_{0}$, is case dependent. From Table 3 , it seems clear that the two Atlantic TCs benefited less from assimilating the QSCAT/ASCAT information. It also is clear that a successful simulation of the TC genesis and early development stages $[54,55]$ is critical requirement for accurate subsequent track prediction.

The Eliassen-Palm (E-P) flux has been widely used in large-scale dynamics. The extension of this concept of eddy momentum (the horizontal component of E-P flux) and heat (the vertical component of E-P flux) in the rotating TC environment [56-58] was successful in explaining vortex Rossby wave-mean flow (azimuthal or tangential winds) interactions. Here, the definitions of the E-P flux and its divergence in the cylindrical isentropic coordinates moving with the rotating TC are the same as in (2) of Chen and Yau [58].

$$
\begin{gathered}
F=\left[-r \overline{(\sigma u)^{\prime} v^{\prime}} ; \overline{\frac{p^{\prime}}{g} M_{\lambda}^{\prime}}\right], \\
\nabla \cdot F=\frac{1}{r} \frac{\partial}{\partial r}\left[-r^{2} \overline{(\sigma u)^{\prime} v^{\prime}}\right]+\frac{\partial}{\partial \theta} \overline{\left[\frac{p^{\prime}}{g} M_{\lambda}^{\prime}\right]},
\end{gathered}
$$

where the overbars denote Eulerian azimuthal means. The primes are deviations against the azimuthal means. Variables $u$ and $v$ are, respectively, the radial and tangential winds relative to the moving TC center. $\theta$ is isentrope, $\lambda$ is azimuth, and $r$ is radius. When $\theta, \lambda$, and $r$ are used as subscripts, it means derivative with respective to isentropic, azimuthal, and radial directions. $\sigma$ is the isentropic density, $M$ is Montgomery function, $p$ is air pressure, and $g$ is gravitational acceleration.

The horizontal component is the eddy (anomalous radial winds) transfer of angular momentum (of the azimuthal or radial winds). Specifically, it is the eddy momentum transport in the radial direction but is of opposite sign. Associated with an outward pointing EP-flux eddies transport positive momentum inward and vice versa. The tangential winds here are analogous to the mean zonal winds in large-scale system. Thus, when the divergence of E-P is positive, eddies tend to accelerate the mean tangential winds; and when negative, the tangential winds abate. It has been shown that this divergence also is directly proportional to the radial eddy PV flux [57]. The nonacceleration theorem [59-62] in such circumstances means that the divergence is negligible when the TC is at a symmetrically steady developing stage.

Here we performed E-P flux analyses for the interested TCs based on the atmospheric parameters from EXP1s. The E-P flux $[56,58,63]$ and its divergence in the Eulerian mean equations, expressed in isentropic coordinates (Equations (2)), are used to explore the wave dynamics and wave-mean flow interactions. The E-P flux, or the vortex Rossby waverelated eddy momentum and heat transport, varies as a response to the evolution of the potential vorticity (PV) structure. The wave-mean flow interaction has a significant effect on the tangential wind, which is dominated by the mean circulation, especially through the asymmetric diabatic heating. During genesis and the intensification phase, the asymmetric diabatic heating, through the waves-base flow interaction, tends to enhance the mean circulation. For example, the radially interlaced positive and negative E-P flux divergence bands at a lower level $(288 \mathrm{~K})$ correspond well with the convective bands (Figure 4). As indicated in Figure 4, during the entire TC life cycle, the E-P flux first increases but then decreases after the TC's circulation becomes completely circular. The magnitude and (time) interval of its persistence are directly related to TC severity. TC Katrina is much stronger compared to Ketsana because it has an extended period of positive contribution from the E-P flux (compare Figures 4 and 5).

The vertical component of the E-P flux (here it is in isentropic coordinates) is proportional to eddy transport of heat $\left(\mathrm{kg} \mathrm{K}^{-1} \mathrm{~s}^{-2}\right)$. The numerical value of the vertical component is orders of magnitude smaller and has to be properly 

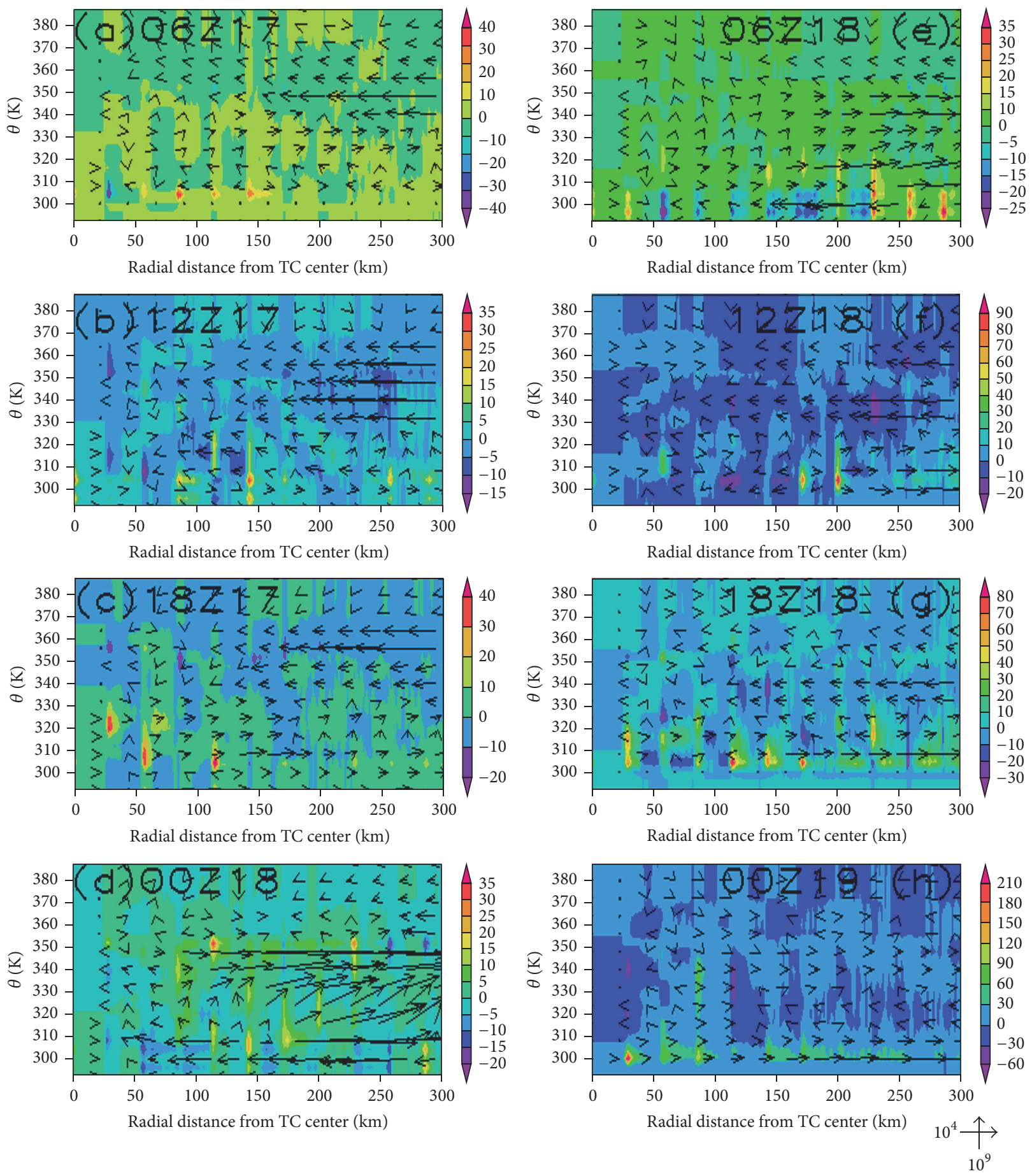

Figure 4: Eliassen-Palm flux and its divergence in isentropic coordinates valid at 8 times for figures (a)-(h) at 6-hr intervals. The horizontal and vertical components of E-P fluxes have units of $\mathrm{kg} \mathrm{m} \mathrm{K}^{-1} \mathrm{~s}^{-2}$ and $\mathrm{kg} \mathrm{s}^{-2}$, respectively. The shades for the divergence of the E-P flux have 5 $\times 10^{3} \mathrm{~kg} \mathrm{~K}^{-1} \mathrm{~s}^{-2}$, with positive regions shaded with warm colors. The horizontal axis indicates the distance from the storm center. Only values within a $300 \mathrm{~km}$ radius of the storm center are shown.

scaled in the plots (Figure 6). Except for regions of strong upward motion (e.g., the eyewall at the intensification stage), the vertical component remains small. Unlike the situation in large-scale dynamics, the divergence contribution from the vertical component also is smaller than the horizontal component. From the vertical component of the divergence contribution, however, the TC genesis stage generally has three layers. These are temporally rather stable, unlike the chaotic horizontal component divergence contribution, which are the near-surface inward flux, the midlevel (PBL top to the $330 \mathrm{~K}$ level) outward flux, and the upper level (above $\sim 340 \mathrm{~K}$ ) inward flux again. This means that the evaporated 

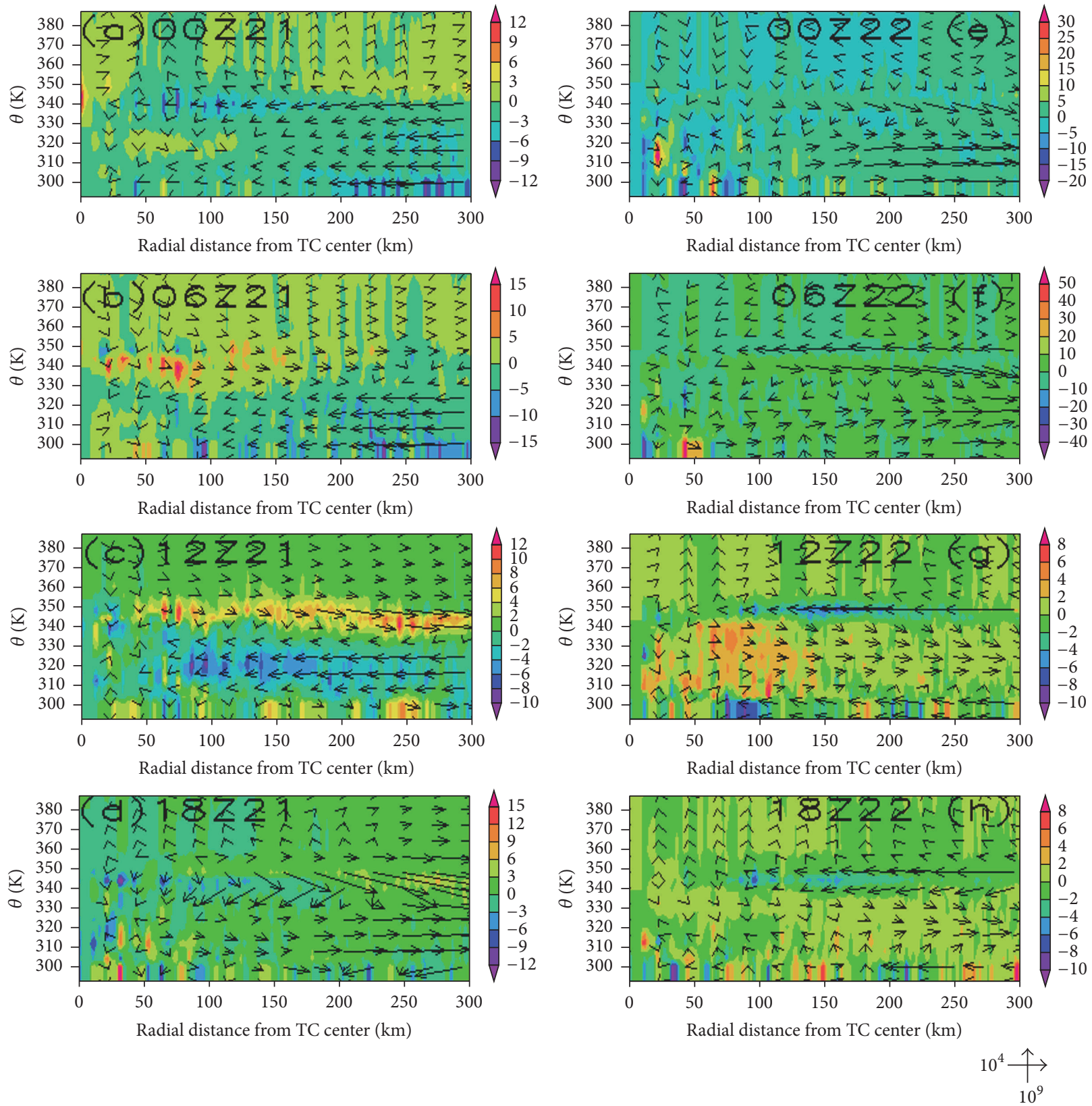

FIgURE 5: As in Figure 4 but for TC Katrina (2005).

moisture from ocean surface is condensed at the middle level. The heat released increases the kinetic energy of the air mass and this kinetic energy is transferred both upward and downward. As a result, the azimuthal winds of the entire air column are accelerated. Because the ultimate energy source is surface evaporation, the development of the TC signifies a net vertical upward energy transport. It is notable that, as the TC expands radially outward, there are alternating accelerating and deaccelerating bands, corresponding closely to the convective rain bands. Merging or further downward cascading is a response to diabatic heating and environmental flow vorticity (shear) interaction as well as lower boundary roughness changes. These stages usually signify intensity changes, as reflected in the total kinetic energy [64]. In view of the errors of the short-range tracking and intensity forecast (i.e., the maximum $10 \mathrm{~m}$ wind speeds have the largest errors) and recent work by Montgomery et al. [42], it is argued here that remote sensing information at the rapid intensification stage should be highly informative.

Katrina just experienced a transition at 12 UTC on 23 August (for brevity, the E-P flux figure is not shown). Assimilating surface winds at this time is most informative and improves the prediction skill significantly (Figure 7). Figure 7 illustrates the improvements in track forecasting for Katrina, using the data assimilation procedures described above. In this case and the remaining three cases, assimilating more 


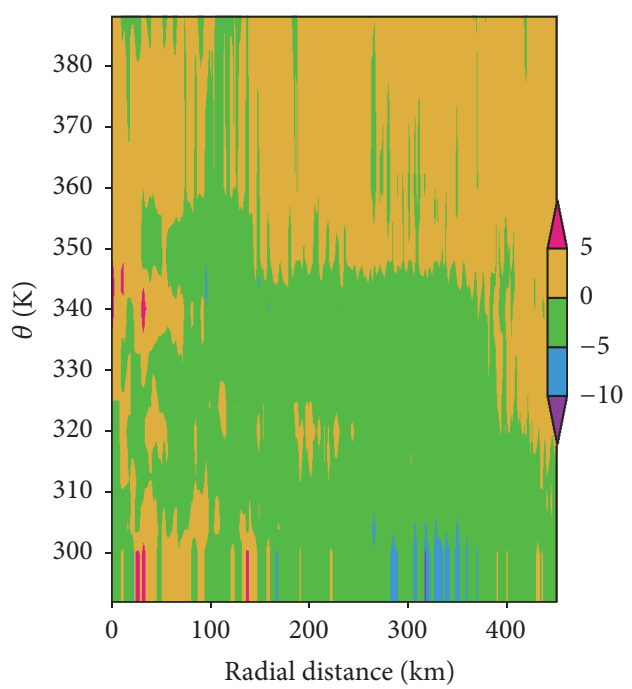

(a)

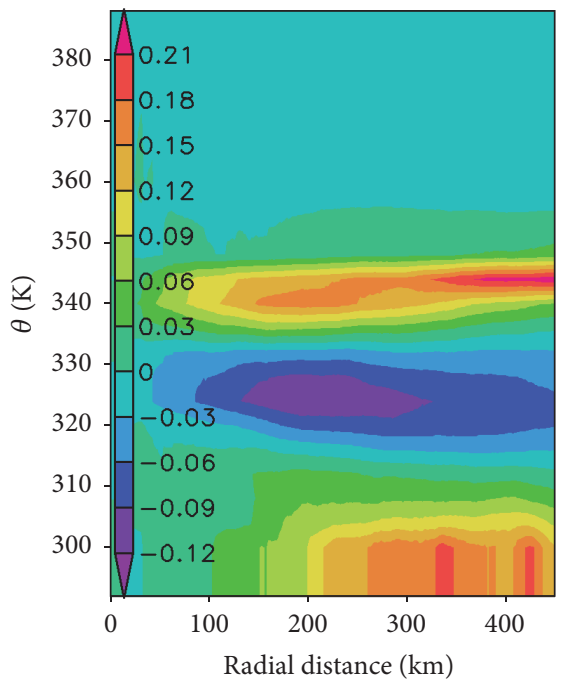

(b)

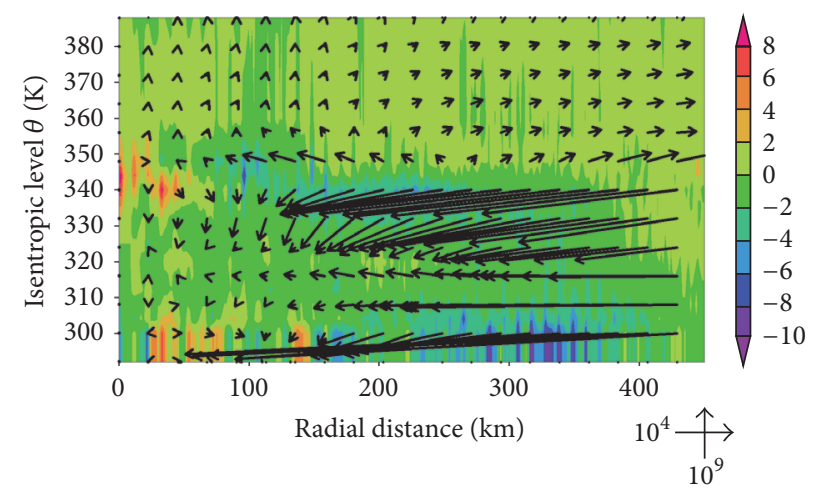

(c)

Figure 6: Divergence of the E-P flux. (a) is the contribution from the horizontal component (eddy transfer of momentum), (b) is the vertical (eddy transfer of heat) component, and (c) is the total for TC. Katrina at 00 UTC on 21 August 2005.

information always results in improvements in track prediction (see Table 3). The degree of improvement, however, is case dependent and difficult to generalize. The added benefit of assimilating QSCAT near-surface winds is relatively insignificant compared to similar cases in the Atlantic or Pacific TC basins. The reasons may be multifaceted and here an explanation is provided from E-P flux analysis perspective. From the above discussion, it is apparent that the genesis and early developmental stages are difficult for the numerical simulation of TCs. For example, incomplete information on flow vectors may result in location errors in the identified cyclonic circulation center. In the above discussion, E-P flux analysis was performed in a regional area (defined by red circles in Figure 1). The center of this circular region is translated along the IBTrACS path and the E-P fluxes are estimated continually at 6-hr intervals. Accordingly, a time series of E-P flux divergence is obtained, averaged over the circular regional area. The peak value of the divergence is reached at a location identified in Figure 8. Interestingly, for Katrina, the region is almost entirely within the interorbital gap between successive swaths of QSCAT/ASCAT and hence is largely overlooked by satellites. The additional improvement gained using the approach adopted here is entirely from assimilating surface wind information beyond the more informative region. Otherwise, the improvement from assimilating QSCAT observations would be even more significant. This finding agrees with Singh et al. [65] that realized the importance of the satellite overpass at the time of model initialization. They also concluded that if cyclone falls in the orbital gaps then there is very lower chance of improvement, particularly in intensity, in the TC prediction, even if the satellite pass is available in the vicinity of the TCs. The other three cases (Figure 8) show more benefit, especially Typhoon Megi. The near-surface cyclonic wind shear zone in this case is traced by ASCAT. As a result, the improvement of the track simulation, from assimilating near-surface winds, is the most significant among the cases studied (Table 3 ). The improvements gained from assimilating satellite data also are reflected in the decrease of the cost function (Figure 9). For all combinations of assimilating SSM/I TPW and QSCAT winds, the case of Ketsana outperforms the case with Katrina. There are important potential implications for future satellite mission planning for 


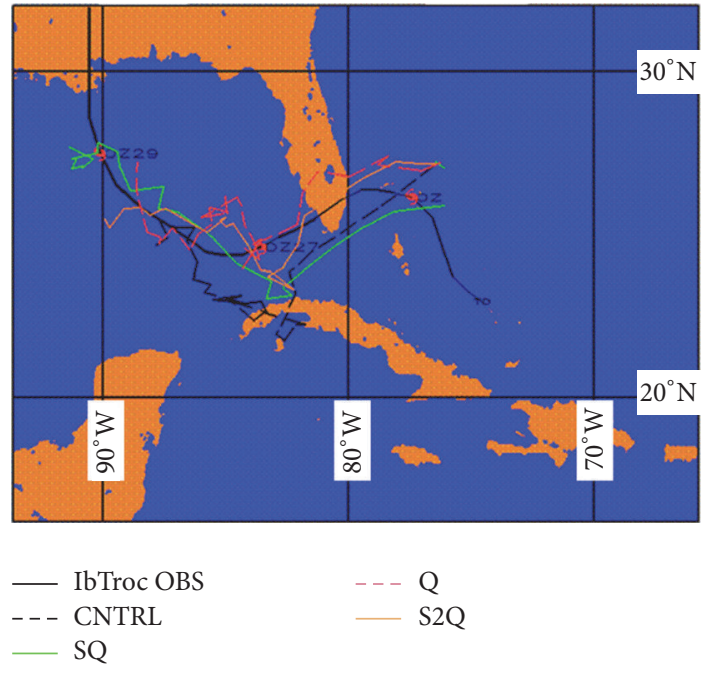

FIGURE 7: Improvement of track simulations of Katrina (2005) through assimilating SSM/I and QSCAT observations. Model simulated tracks for Hurricane Katrina commencing at 00 UTC on 25 August 2005. The black dashed line is the forecast control case. The solid black line is best track data; the green line represents the case of assimilating SSM/I and one QSCAT. Assimilation of one QSCAT surface wind vector field is shown in red, and the one assimilation of SSMI and two QSCAT observations are shown in brown.

TC research. Gaps in orbital coverage over the TC genesis region must be limited. This is achievable either by using multiple polar-orbiting satellites or by deploying geostationary systems directly over the regions of interest (e.g., the proposed geostationary satellites over Western Australia). Comparatively, the synergy of the data from different scatterometers/radiometers would be technically less demanding. For example, our next stage of study would focus on using scatterometer on Oceansat-II (launched by the Indian Space Research Organization), which has much wider swath $(\sim 1800 \mathrm{~km})$ than ASCAT, together with ASCAT for simulating TCs after 2009.

To demonstrate how assimilation affects TC forecasts, Figure 10 shows the 60-hour track forecasts for Ketsana initialized at 00 UTC on 16 October. Generally, there are progressive improvements in track forecast skill from EXP2 to EXP4. EXP4, which assimilated the most information, also produced the most accurate forecasts. As the genesis basin is ideally located for QSCAT observations, the improvements from forecast experiment EXP2 over the CNTRL case are the most significant among the four cases. The use of rmse to quantify track improvement provides the geographical closeness of the tracks. In the case of Ketsana (Figure 10), it is clear that the model predictions from assimilation schemes EXP2 and EXP3 both closely follow the observed tracks. The assimilation scheme EXP2 has a larger temporal error (because the system evolved slower than reality) than EXP3. Thus, the track plots in Figure 7 need to be interpreted with reference to Table 3 to determine the additional gain from assimilating remotely sensed data.

\section{Summary}

Variational data assimilation of satellite data was identified as key for the greatly improved forecast skill of NWPs after 1999 [66]. To investigate the impact of assimilating the QSCAT/ASCAT surface wind observations and total precipitable water from the SSM/I in the WRF on the track forecasting of four tropical cyclones over the global basins, three data denial experiments for each TC are performed which progressively assimilate SSM/I and QSCAT information. Compared to the control case, in which only traditional GFS analysis atmospheric parameters provide the lateral boundary conditions and initialize the model run, the data assimilation runs performed consistently better with the additional input information. However, it is important to emphasize that the improvement was case dependent. Asymmetric transport of thermal energy and momentum in the form of wave-base flow (azimuthal winds) interactions are critical for TC genesis and intensification. E-P fluxes defined in a storm-following cylindrical coordinate system (origin at the storm center) are suitable indicators of storm development. For each TC, E-P fluxes are calculated within a circular region of radius of $300 \mathrm{~km}$, defined as the region of influence. The center of the region of influence is then translated along the IBTrACS storm track, 6 hours apart. The divergence of E-P fluxes produces a time series. During the TC life cycle, this time series first increases in magnitude and then decreases to negligible values when the TC is fully developed and has become circular. The region of influence corresponds to the peak value of E-P flux divergence. If the TC's physical extent is fully covered at this time by the polar-orbiting satellites, numerical forecasting of the corresponding storm will profit most from assimilating remotely sensed information. Conversely, if the critical genesis region lies within the interorbital gaps of the satellite swaths then, independently of how strong the TC becomes subsequently, the improvement from assimilating near-surface winds and total precipitable water is limited. This confirms the need for increased satellite reconnaissance of high interest regions to enhance the skill of TC genesis and track predictions. The fact that the denial of QSCAT/ASCAT data introduces large time and location errors in the WRF forecast of TC Ketsana 2003 and Megi 2010's tracks illustrates the importance of the availability of near-surface winds for accurate TC prediction. It also agrees with Žagar et al. [11] who concluded that, in tropical regions, wind information is more efficient at recovering equatorial waves than are mass fields. Thus, satellites provide vital information on the largescale atmospheric condition over areas that would otherwise be sparsely observed. Although the level of improvement is not the same in all forecasts, the sensitivity is salient and clearly demonstrates the value of employing an advanced data assimilation algorithm that uses flow-dependent error covariance.

In this study, the WRF model included the improvement of surface latent heat fluxes described in [32]. The control runs still have significant discrepancies compared with the best track data. Further improvements of forecasting skill came from the data assimilation scheme and the additional 


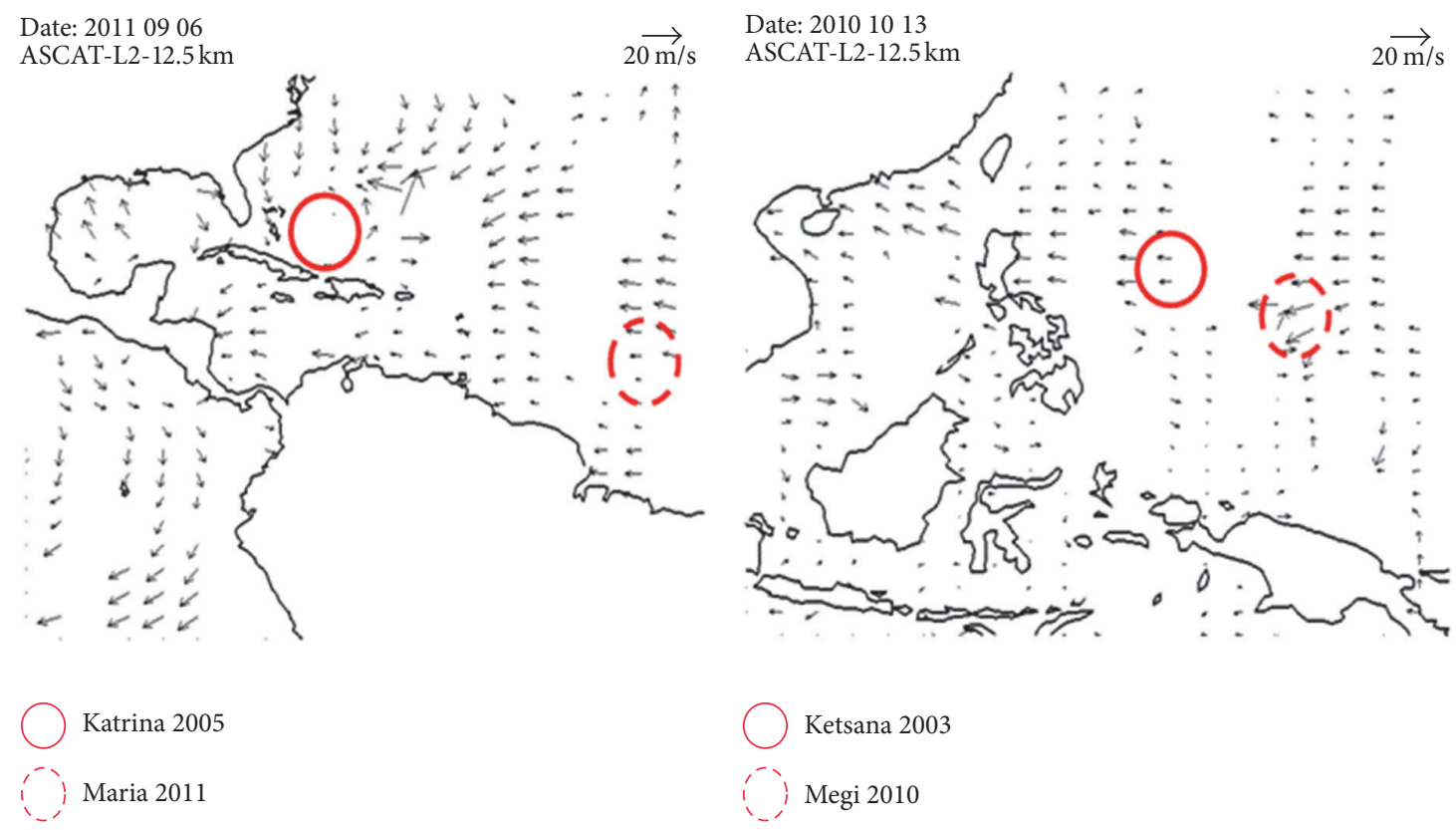

(a)

(b)

FIgURE 8: Identified regions (red circles) show that E-P fluxes contribute most strongly to the azimuthal circulation of TC. The ASCAT observed flow shows the satellite coverage over the TC basins. It is clear from (a) that Katrina's critical development region is missed whereas in (b) Megi's critical development region is well-captured. TCs Maria in (a) and Ketsana in (b) are partially covered by the satellite observations.

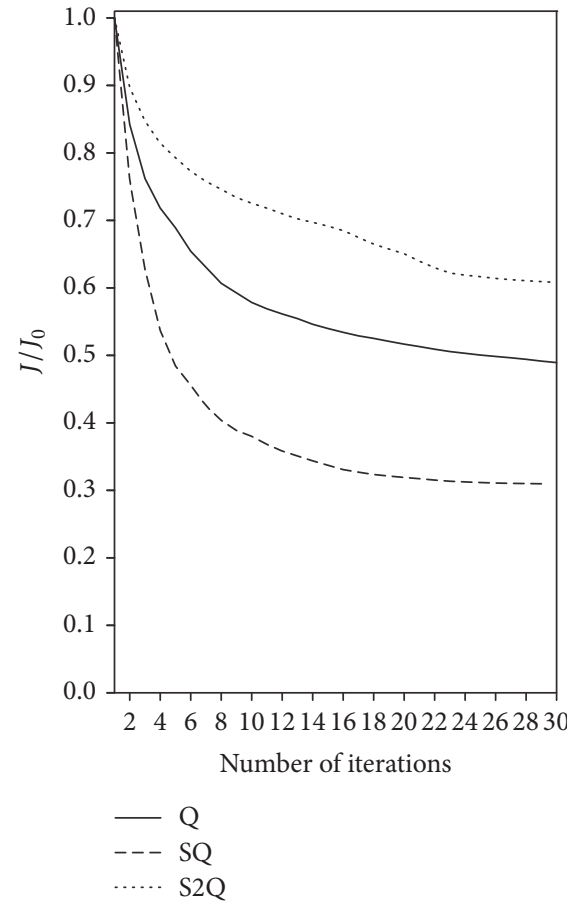

(a)

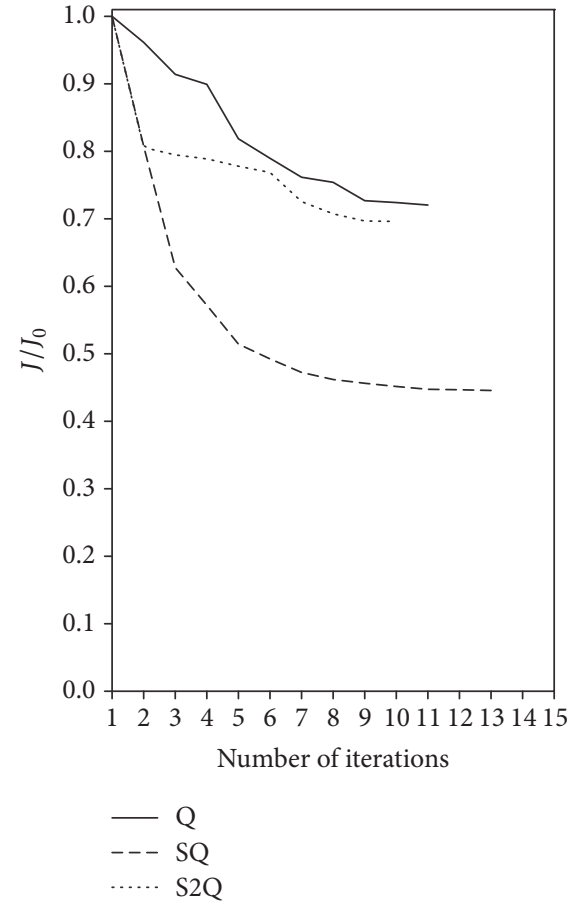

(b)

Figure 9: Decrease of cost functions (normalized by the initial cost function values $J_{0}$ ), (a) for Ketsana 2003 and (b) for Katrina 2005. Comparisons among Q, SQ, and S2Q within each TC case are not preferred because the cost functions are of different structure and of very different initial values. For Ketsana, the decrease of cost function is more efficient than that for Katrina, for all three cases of assimilating remotely sensed information. 


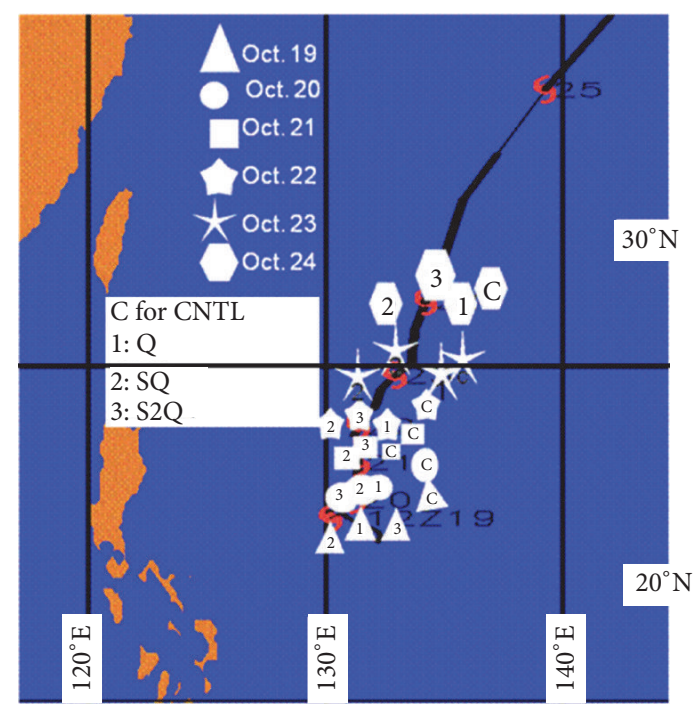

Figure 10: Pacific basin tracks for Typhoon Ketsana (2003) commencing 12 UTC on 19 October 2003. The control (no satellite data) forecast case is labelled " $\mathrm{C}$ "; the black line is the best track. Symbol "1" identifies the case that assimilated only QSCAT; "2" shows the case that assimilated SSM/I and one level of QSCAT; and " 3 " is the case that assimilated SSM/I and two sequential sets of QSCAT observations. Different markers are used to illustrate temporal errors in track prediction. Triangles represent 12 UTC on 19 October; circles represent 12 UTC on 20 October; squares represent 12 UTC on 21 October; blunt pentagons represent 12 UTC on 22 October; acute pentagons represent 12 UTC on 23 October; and hexagons represent 12 UTC on 24 October. Two tracks can be close geographically but with significant phase errors (e.g., the control case " $C$ " leads while the case " 1 " that assimilated QSCAT lags behind the IBTrACS).

remotely sensed observations. Although this is a viable approach and is the best choice for a given version of WRF, it is clear that model improvements, especially in the physical parameterization, need to be pursued to further enhance forecasting skill [66]. In future research, a version of WRF will be used that includes an interactive sea-surface temperature. TCs produce strong currents that vertically mix the nearsurface ocean water, frequently to well below the regional mixed layer depth and cause upwelling of cooler water to the surface. The ensuing reduction in surface evaporation usually is significant and may have a decisive impact on the patterns and totals of extreme precipitation and also on wind energy distribution. This effect is especially strong when TCs approach land [67-69].

\section{Additional Points}

The data used in this research all are publicly available: SST data are available at ftp://polar.ncep.noaa.gov/pub/history/ sst/; SSMI data are available at ftp://ftp.ssmi.com/qscat and $\mathrm{ftp} / / / \mathrm{ftp} . s s m i . c o m / s e a w i n d s ;$ and the meteorological parameters are obtainable from http://rda.ucar.edu/datasets/ds$083.2 \%$

\section{Competing Interests}

The author declares that there is no conflict of interests regarding the publication of this paper.

\section{Acknowledgments}

The author is grateful to the following colleagues for discussion on variational data assimilation and on what satellite data already were included in the final analysis: Dr. Grace Peng, Professor Ming Xue, Dr. P. Dong, Dr. J. Dong, Dr. X. B. Wang, Dr. J. LeMarshall, Dr. G. P. Zhang, and Dr. J. Kepert.

\section{References}

[1] H. Kunreuther and E. Michel-Kerjan, At War with the Weather: Managing Large-Scale Risks in a New Era of Catastrophies, MIT Press, New York, NY, USA, 2009.

[2] J. M. Done, G. J. Holland, C. L. Bruyère, L. R. Leung, and A. Suzuki-Parker, "Modeling high-impact weather and climate: lessons from a tropical cyclone perspective," Climatic Change, vol. 129, no. 3-4, pp. 381-395, 2015.

[3] N. Mori, M. Kato, S. Kim et al., "Local amplification of storm surge by Super Typhoon Haiyan in Leyte Gulf," Geophysical Research Letters, vol. 41, no. 14, pp. 5106-5113, 2014.

[4] B. Xiang, S.-J. Lin, M. Zhao et al., "Beyond weather time-scale prediction for hurricane sandy and super typhoon haiyan in a global climate model," Monthly Weather Review, vol. 143, no. 2, pp. 524-535, 2015.

[5] Y. Wang, Y. Wang, and H. Fudeyasu, "The role of Typhoon Songda (2004) in producing distantly located heavy rainfall in Japan," Monthly Weather Review, vol. 137, no. 11, pp. 3699-3716, 2009.

[6] G. Villarini, D. A. Lavers, E. Scoccimarro et al., "Sensitivity of tropical cyclone rainfall to idealized global-scale forcings," Journal of Climate, vol. 27, no. 12, pp. 4622-4641, 2014.

[7] J. Kam, J. Sheffield, X. Yuan, and F. E. Wood, “The influence of atlantic tropical cyclones on drought over the Eastern United States (1980-2007)," Journal of Climate, vol. 26, no. 10, pp. 30673086, 2013.

[8] Y. Han, P. van Delst, Q. Liu et al., "Community radiative transfer model (CRTM): version 1,” NOAA Tech. Rep 122, 2006.

[9] J. Le Marshall, L. Uccellini, F. Einaudi, and J. G. Yoe, "The joint center for satellite data assimilation," Bulletin of the American Meteorological Society, vol. 88, no. 3, pp. 329-340, 2007.

[10] X. Zou, F. Weng, B. Zhang, L. Lin, Z. Qin, and V. Tallapragada, "Impacts of assimilation of ATMS data in HWRF on track and intensity forecasts of 2012 four landfall hurricanes," Journal of Geophysical Research Atmospheres, vol. 118, no. 20, pp. 1155811576, 2013.

[11] N. Žagar, N. Gustafsson, and E. Källén, "Variational data assimilation in the tropics: The impact of a background-error constraint," Quarterly Journal of the Royal Meteorological Society, vol. 130, no. 596, pp. 103-125, 2004.

[12] L. M. McMillin and H. E. Fleming, "Atmospheric transmittance of an absorbing gas: a computationally fast and accurate transmittance model for absorbing gases with constant mixing ratios in inhomogeneous atmospheres," Applied Optics, vol. 15, no. 2, pp. 358-363, 1976.

[13] R. W. Saunders, M. Matricardi, and P. Brunel, "An improved fast radiative transfer model for assimilation of satellite radiance 
observations," Quarterly Journal of the Royal Meteorological Society, vol. 125, no. 556, pp. 1407-1425, 1999.

[14] S.-H. Chen, "The impact of assimilating SSM/I and QuikSCAT satellite winds on Hurricane Isidore simulations," Monthly Weather Review, vol. 135, no. 2, pp. 549-566, 2007.

[15] R. Singh, P. K. Pal, C. M. Kishtawal, and P. C. Joshi, "The impact of variational assimilation of SSM/I and quickSCAT satellite observations on numerical simulation of Indian ocean tropical cyclones," Weather and Forecasting, vol. 23, no. 3, pp. 460-476, 2008.

[16] N. Bassill, "Accuracy of early GFS and ECMWF Sandy (2012) track forecasts: evidence for a dependence on cumulus parameterization," Geophysical Research Letters, vol. 41, no. 9, pp. 32743281, 2014.

[17] T. Mcnally, M. Bonavita, and J.-N. Thépaut, "The role of satellite data in the forecasting of hurricane sandy," Monthly Weather Review, vol. 142, no. 2, pp. 634-646, 2014.

[18] Y. Zhu, D. Hou, X. Zhou, R. Wobus, M. Pena, and J. Peng, "The development of Global Ensemble Forecast System (GEFS)," in Proceedings of the 26th Conference on Weather Analysis and Forecasting/22nd Conference on Numerical Weather Prediction, American Meteorological Society, Atlanta, Ga, USA, February 2014, https://ams.confex.com/ams/94Annual/webprogram/ Paper236915.html.

[19] W. C. Skamarock, J. B. Klemp, J. Dudhia et al., "A description of the advanced research WRF version 3," NCAR Tech. Note NCAR/TN-475+STR, 2008.

[20] J. Figa-Saldaña, J. J. W. Wilson, E. Attema, R. Gelsthorpe, M. R. Drinkwater, and A. Stoffelen, "The advanced scatterometer (ASCAT) on the meteorological operational (MetOp) platform: a follow on for European wind scatterometers," Canadian Journal of Remote Sensing, vol. 28, no. 3, pp. 404-412, 2002.

[21] F. J. Wentz, "SSM/I version-7 calibration report," Report 011012, Remote Sensing Systems, Santa Rosa, Calif, USA, 2013.

[22] K. R. Knapp, M. C. Kruk, D. H. Levinson, H. J. Diamond, and C. J. Neumann, "The international best track archive for climate stewardship (IBTrACS)," Bulletin of the American Meteorological Society, vol. 91, no. 3, pp. 363-376, 2010.

[23] R. Houze Jr., "Clouds in tropical cyclones," Monthly Weather Review, vol. 138, no. 2, pp. 293-344, 2010.

[24] E. A. Ritchie and G. J. Holland, "Large-scale patterns associated with tropical cyclogenesis in the western Pacific," Monthly Weather Review, vol. 127, no. 9, pp. 2027-2043, 1999.

[25] J. R. Eyre, G. A. Kelly, A. P. McNally, E. Andersson, and A. Persson, "Assimilation of TOVS radiance information through onedimensional variational analysis," Quarterly Journal-Royal Meteorological Society, vol. 119, no. 514, pp. 1427-1463, 1993.

[26] J. C. Derber and W.-S. Wu, "The use of TOVS cloud-cleared radiances in the NCEP SSI analysis system," Monthly Weather Review, vol. 126, no. 8, pp. 2287-2299, 1998.

[27] K. Ide, P. Courtier, M. Ghil, and A. C. Lorenc, "Unified notation for data assimilation: operational, sequential and variational," Journal of the Meteorological Society of Japan, vol. 75, no. 1, pp. 181-189, 1997.

[28] J. R. Eyre, "A bias correction scheme for simulated TOVS brightness temperatures," ECMWF Tech. Memo 186, 1992.

[29] A. C. Lorenc, "Analysis methods for numerical weather prediction," Quarterly Journal of the Royal Meteorological Society, vol. 112, no. 474, pp. 1177-1194, 1966.

[30] D. F. Parrish and J. C. Derber, "The National Meteorological Center's spectral statistical-interpolation analysis system," Monthly Weather Review, vol. 120, no. 8, pp. 1747-1763, 1992.
[31] D. P. Jorgensen, "Mesoscale and convective-scale characteristics of mature hurricanes. Part II. Inner core structure of hurricane allen (1980)," Journal of the Atmospheric Sciences, vol. 41, no. 8, pp. 1287-1311, 1984.

[32] D. Ren, M. J. Lynch, L. M. Leslie, and J. LeMarshall, "Sensitivity of tropical cyclone tracks and intensity to ocean surface temperature: four cases in four different basins," Tellus A, vol. 66, Article ID 24212, 2014.

[33] S.-Y. Hong, J. Dudhia, and S.-H. Chen, "A revised approach to ice microphysical processes for the bulk parameterization of clouds and precipitation," Monthly Weather Review, vol. 132, no. 1, pp. 103-120, 2004.

[34] Y. Noh, W. G. Cheon, S. Y. Hong, and S. Raasch, "Improvement of the K-profile model for the planetary boundary layer based on large eddy simulation data," Boundary-Layer Meteorology, vol. 107, no. 2, pp. 401-427, 2003.

[35] J. S. Kain, “The Kain-Fritsch convective parameterization: an update," Journal of Applied Meteorology, vol. 43, pp. 170-181, 2004.

[36] E. J. Mlawer, S. J. Taubman, P. D. Brown, M. J. Iacono, and S. A. Clough, "Radiative transfer for inhomogeneous atmospheres: RRTM, a validated correlated-k model for the longwave," Journal of Geophysical Research D: Atmospheres, vol. 102, no. 14, pp. 16663-16682, 1997.

[37] J. Dudhia, "Numerical study of convection observed during the Winter Monsoon Experiment using a mesoscale two-dimensional model," Journal of the Atmospheric Sciences, vol. 46, no. 20, pp. 3077-3107, 1989.

[38] M. B. Ek, K. E. Mitchell, Y. Lin et al., "Implementation of Noah land surface model advances in the National Centers for Environmental Prediction operational mesoscale Eta model," Journal of Geophysical Research: Atmospheres, vol. 108, no. 22, 2003.

[39] E. Kalnay, M. Kanamitsu, R. Kistler et al., "The NCEP/NCAR 40-year reanalysis project," Bulletin of the American Meteorological Society, vol. 77, no. 3, pp. 437-471, 1996.

[40] W. H. Schubert, M. T. Montgomery, R. K. Taft et al., "Polygonal eyewalls, asymmetric eye contraction, and potential vorticity mixing in hurricanes," Journal of the Atmospheric Sciences, vol. 56, no. 9, pp. 1197-1223, 1999.

[41] H.-C. Kuo, R. T. Williams, and J.-H. Chen, "A possible mechanism for the eye rotation of Typhoon Herb," Journal of the Atmospheric Sciences, vol. 56, no. 11, pp. 1659-1673, 1999.

[42] M. T. Montgomery, M. M. Bell, S. D. Aberson, and M. L. Black, "Hurricane Isabel (2003): new insights into the physics of intense storms. Part I: mean vortex structure and maximum intensity estimates," Bulletin of the American Meteorological Society, vol. 87, no. 10, pp. 1335-1347, 2006.

[43] J. Done, C. A. Davis, and M. Weisman, “The next generation of NWP: explicit forecasts of convection using the weather research and forecasting (WRF) model," Atmospheric Science Letters, vol. 5, no. 6, pp. 110-117, 2004.

[44] J. Chan and W. M. Gray, "Tropical cyclone movement and surrounding flow relationships," Monthly Weather Review, vol. 110, no. 10, pp. 1354-1374, 1982.

[45] M. Flatau, W. H. Schubert, and D. E. Stevens, "The role of baroclinic processes in tropical cyclone motion: the influence of vertical tilt," Journal of the Atmospheric Sciences, vol. 51, no. 18, pp. 2589-2601, 1994.

[46] J. Chan, "The physics of tropical cyclone motion," Annual Review of Fluid Mechanics, vol. 37, pp. 99-128, 2005. 
[47] L. M. Leslie and G. J. Holland, "On the bogussing of tropical cyclones in numerical models: a comparison of vortex profiles," Meteorology and Atmospheric Physics, vol. 56, no. 1-2, pp. 101110, 1995.

[48] Chun-Chieh Wu and K. A. Emanuel, "Interaction of a baroclinic vortex with background shear: application to hurricane movement," Journal of the Atmospheric Sciences, vol. 50, no. 1, pp. 6276, 1993.

[49] J. C. L. Chan, F. M. F. Ko, and Y. M. Lei, "Relationship between potential vorticity tendency and tropical cyclone motion," Journal of the Atmospheric Sciences, vol. 59, no. 8, pp. 1317-1336, 2002.

[50] G. J. Holland, "Tropical cyclone motion: a comparison of theory and observation," Journal of the Atmospheric Sciences, vol. 41, no. 1, pp. 68-75, 1984.

[51] L. J. Shapiro, "The motion of Hurricane Gloria: a potential vorticity diagnosis," Monthly Weather Review, vol. 124, no. 11, pp. 2497-2508, 1996.

[52] L. J. Shapiro and J. L. Franklin, "Potential vorticity asymmetries and tropical cyclone motion," Monthly Weather Review, vol. 127, no. 1, pp. 124-131, 1999.

[53] L. Wu and B. Wang, "Assessing impacts of global warming on tropical cyclone tracks," Journal of Climate, vol. 17, no. 8, pp. 1686-1698, 2004.

[54] D. S. Nolan, "What is the trigger for tropical cyclogenesis?" Australian Meteorological Magazine, vol. 56, no. 4, pp. 241-266, 2007.

[55] D. S. Nolan, Y. Moon, and D. P. Stern, “Tropical cyclone intensification from asymmetric convection: energetics and efficiency," Journal of the Atmospheric Sciences, vol. 64, no. 10, pp. 33773405, 2007.

[56] W. H. Schubert, "Wave, mean flow interactions and hurricane development," in Proceedings of the 16th Conference on Hurricane and Tropical Meteorology, pp. 140-141, American Meteorological Society, Houston, Tex, USA, May 1985.

[57] J. Molinari, S. Skubis, and D. Vollaro, "External influences on hurricane intensity. Part III: potential vorticity evolution," Journal of the Atmospheric Sciences, vol. 52, pp. 3593-3606, 1995.

[58] Y. Chen and M. K. Yau, "Asymmetric structures in a simulated landfalling hurricane," Journal of the Atmospheric Sciences, vol. 60, no. 18, pp. 2294-2312, 2003.

[59] J. G. Charney and P. G. Drazin, "Propagation of planetary-scale disturbances from the lower into the upper atmosphere," Journal of Geophysical Research, vol. 66, no. 1, pp. 83-109, 1961.

[60] J. G. Charney and A. Eliassen, "On the growth of the hurricane depression," Journal of the Atmospheric Sciences, vol. 21, no. 1, pp. 68-75, 1964.

[61] D. G. Andrews and M. E. McIntyre, "Planetary waves in horizontal and vertical shear. (II). Asymptotic theory for equatorial waves in weak shear," Journal of the Atmospheric Sciences, vol. 33, no. 11, pp. 2049-2053, 1976.

[62] J. P. Boyd, "The non-interaction of waves with the zonally averaged flow on a spherical earth and the interrelationships of eddy fluxes of energy, heat and momentum," Journal of the Atmospheric Sciences, vol. 33, no. 12, pp. 2285-2291, 1976.

[63] Y. Chen and M. K. Yau, "Spiral bands in a simulated hurricane. Part I: vortex rossbywave verification," Journal of the Atmospheric Sciences, vol. 58, no. 15, pp. 2128-2145, 2001.

[64] D. Ren, 4DVAR Retrieval of Prognostic Land Surface Model Variables for ARPS, University of Oklahoma, Norman, Okla, USA, 2004.
[65] R. Singh, C. M. Kishtawal, P. K. Pal, and P. C. Joshi, "Assimilation of the multisatellite data into the WRF model for track and intensity simulation of the Indian Ocean tropical cyclones," Meteorology and Atmospheric Physics, vol. 111, no. 3-4, pp. 103119, 2011.

[66] P. Bauer, A. Thorpe, and G. Brunet, "The quiet revolution of numerical weather prediction," Nature, vol. 525, no. 7567, pp. 47-55, 2015.

[67] K. Emanuel, C. DesAutels, C. Holloway, and R. Korty, "Environmental control of tropical cyclone intensity," Journal of the Atmospheric Sciences, vol. 61, no. 7, pp. 843-858, 2004.

[68] C. A. Davis, W. Wang, S. S. Chen et al., "Prediction of landfalling hurricanes with the advanced hurricane WRF model," Monthly Weather Review, vol. 136, no. 6, pp. 1990-2005, 2008.

[69] R. Anthes, Tropical Cyclones: Their Evolution, Structure and Effects, vol. 41 of Meteorological Monographs Series, American Meteorological Society, Boston, Mass, USA, 1982. 

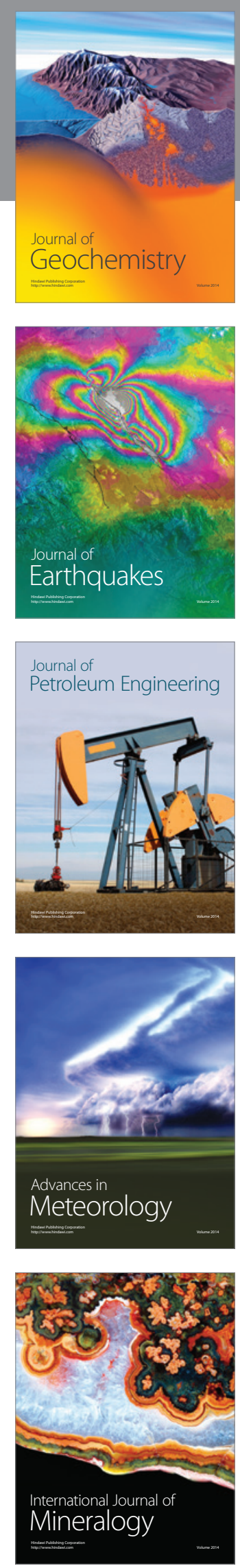
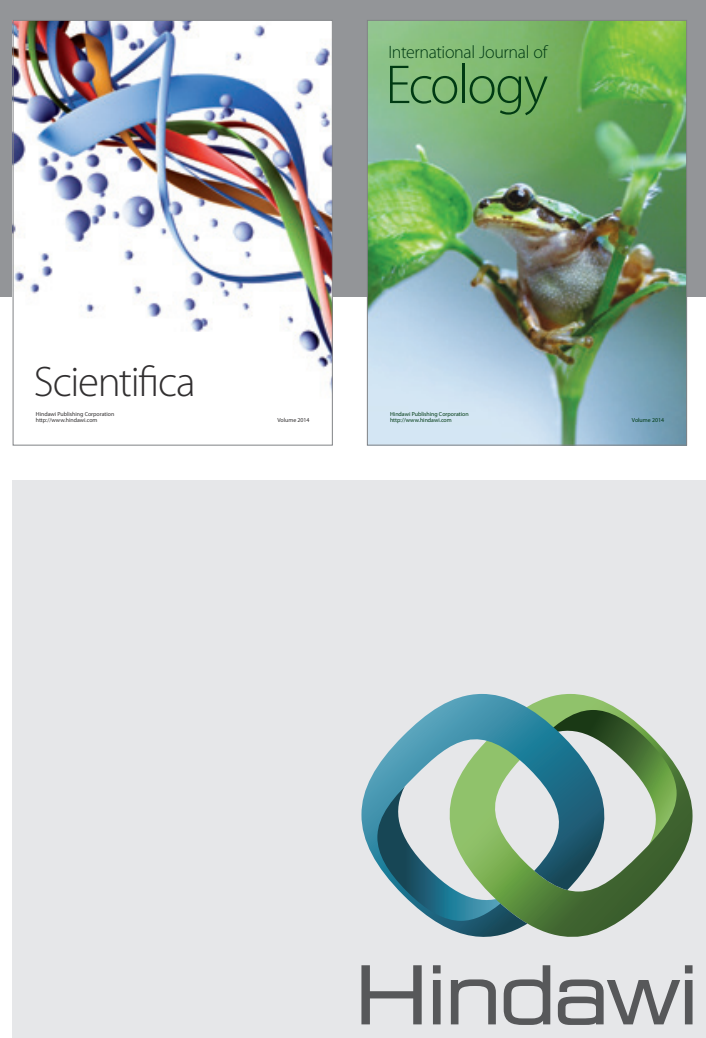

Submit your manuscripts at

http://www.hindawi.com
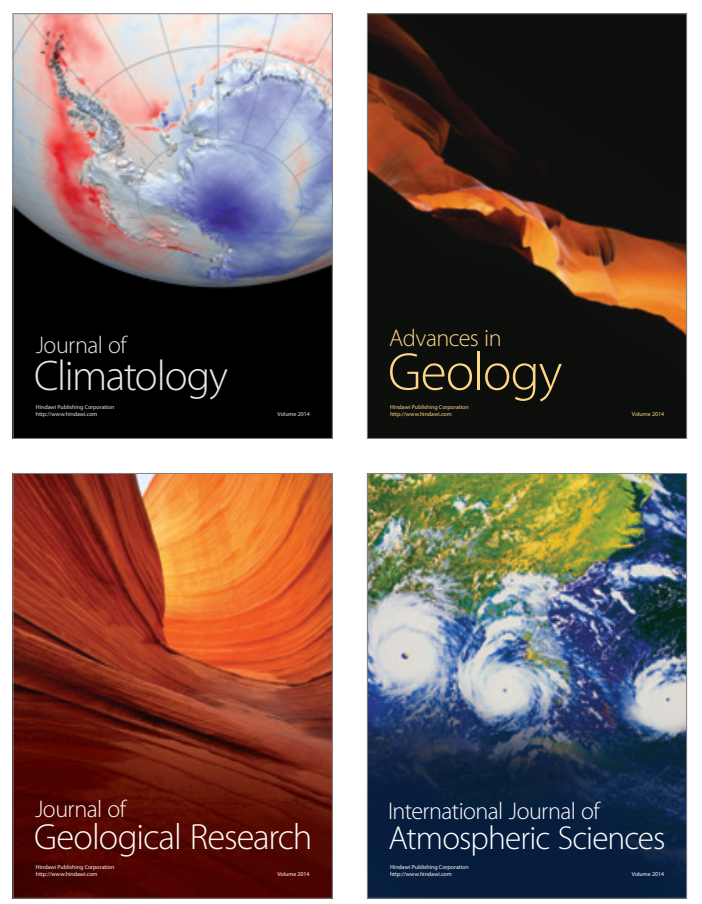

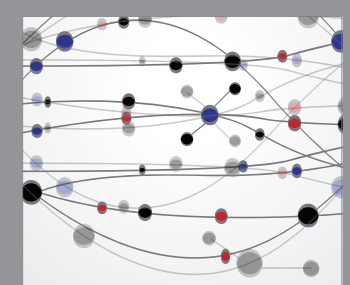

The Scientific

\section{World Journal}
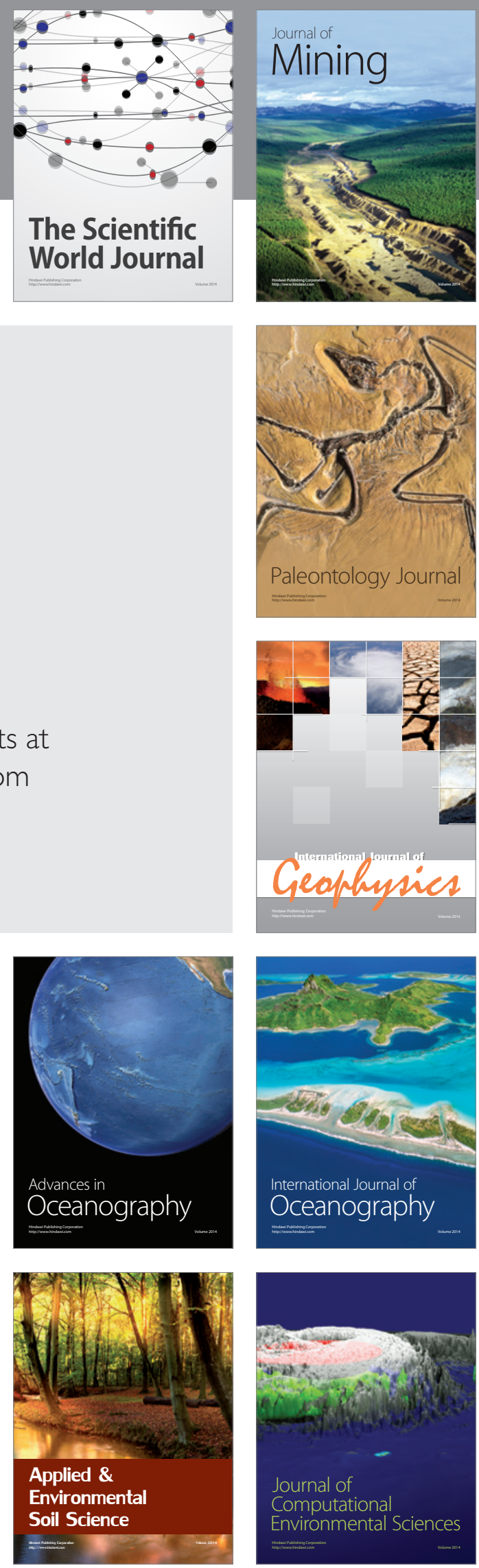